\title{
La Superconductividad: sus orígenes, sus teorías, sus problemas candentes hoy.
}

\author{
Rafael Baquero
}

\author{
Departamento de Física, Cinvestav, San Pedro Zacatenco, Gustavo A. Madero, México D.F.
}

\begin{abstract}
Resumen
En este trabajo doy un breve panorama de los orígenes de la superconductividad, sus características y las teorías que han podido explicar con mucho éxito la llamada superconductividad convencional. Me refiero luego a la superconductividad de alta temperatura crítica y menciono algunos de los problemas de interés en la investigación actual.
\end{abstract}

Superconductivity: discovery, theories and some topics of current research

\begin{abstract}
In this work I briefly refer to the origins of superconductivity, to its characteristics and to the theories that explained very well the so call conventional superconductivity. I further deal with the new high temperature superconductivity and mention briefly some of the problems of interest in the research in the field nowadays.
\end{abstract}

\section{Introducción}

Este artículo está dirigido a un público general pero puede ser útil para personas más familiarizadas con el tema. El 8 de abril de 1911, K. Onnes (1853-1926) descubrió el efecto llamado superconductividad $[1,2]$. La superconductividad se descubrió en metales. Un cristal metálico está compuesto por iones (átomos que han perdido electrones) que vibran alrededor de posiciones determinadas (como los vértices en un cubo) y electrones libres que circulan por el espacio libre entre ellos. Intercambian energía en unidades llamadas fonones (ver abajo). Explicar la física de este fenómeno ha constituido un reto intelectual desde su mismo comienzo.

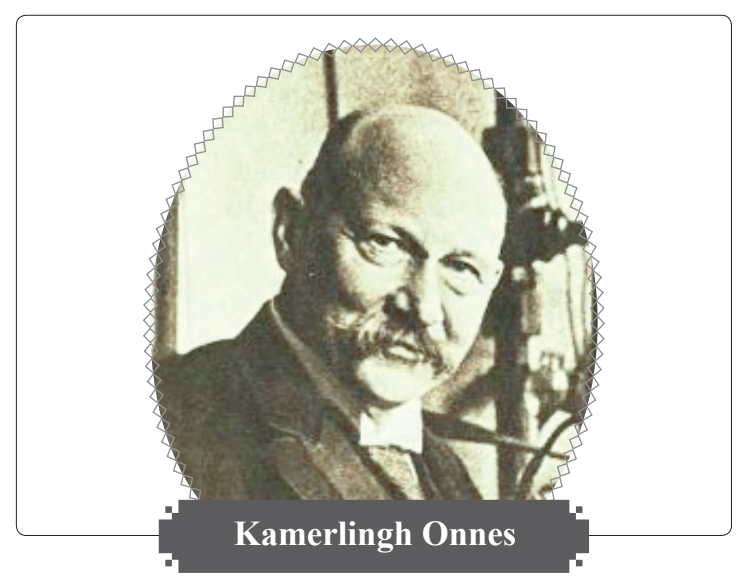

Fue tan solo hacia 1957, a más de 45 años de su descubrimiento, que surgió la primera teoría capaz de dar cuenta de ella. Y es que la superconductividad es un fenómeno cuántico macroscópico que requirió, para su explicación, de la aparición de la Mecánica cuántica ocurrida hacia 1927, de la teoría cuántica del campo, del descubrimiento del Principio de Pauli y de la superficie de Fermi en los metales, hechos que no se conocieron y conjuntaron cabalmente sino hacia la década de los cincuentas del Siglo XX. La aparición del efecto isótopo que consiste en que la temperatura crítica, Tc, más abajo de la cual aparece la superconductividad, depende de la masa del isótopo (átomo idéntico pero con diferente masa). Este hecho indicó el camino para describir el fenómeno como un efecto de interacción entre los electrones y la red (electrón-fonón) descrita por Frölich [3]. Pero fue solo cuando Cooper [4] describió el efecto que una atracción de cualquier origen tiene sobre un par de electrones situados sobre un mar de Fermi ( ver más abajo) que Bardeen comprendió que estaba enfrente de la clave de la superconductividad. Así surgió la Teoría BCS [5] y muy poco después, su extensión, las ecuaciones de Eliashberg [6] que dieron cuenta exacta de todos los superconductores convencionales. En 1986, cuando se celebraban los 75 años

\section{Correspondencia:}

Rafael Baquero, rbaquero@fis.cinvestav.mx

Recibido: 26 de octubre de 2013

Aceptado: 7 de mayo de 2014 
del descubrimiento de la superconductividad, el fenómeno se conocía bien, las aplicaciones tecnológicas posibles habían sido realizadas, esencialmente, $y$, lo bajo de las temperaturas críticas a las cuales el fenómeno aparecía (siempre menores a $23 \mathrm{~K}$ ) determinaban que nuevas aplicaciones fuesen económicamente desventajosas. La superconductividad como tema de estudio, desaparecía. Todo estaba hecho en este campo. No había nada que investigar.

En ese mismo año de 1986, sucedió algo inusitado, tremendo, impresionante. Simplemente, Berdnoz, el asistente de Müller en ese laboratorio, retornó esa tarde, aunque ya quería regresar a casa. Había dejado la medición de la resistividad en función de la temperatura, de un compuesto de lantano. Fue entonces cuando ocurrió la sorpresa. Observó que a $30 \mathrm{~K}^{1}$, aparecía la superconductividad [7]. Increíble! Algo realmente nuevo tenía enfrente. Y así fue. Berdnoz y Müller [8] descubrieron la nueva superconductividad de alta temperatura crítica en el año de 1986 y fueron galardonados con el Premio Nobel en 1987. Poco después, Chu [9] descubrió el YBCO con una Tc de alrededor de los 90K y abrió el camino para el descubrimiento de elementos de una mayor temperatura crítica. Las posibilidades tecnológicas de la superconductividad parecían abrirse camino. Pero, para los teóricos el reto regresó. No sabemos explicar la nueva superconductividad! El reto persiste incluso hoy (noviembre 2014). Esa es la fascinante historia de este peculiar fenómeno.

El interés en la superconductividad viene también por la inmensa cantidad de aplicaciones posibles y ya realizadas. En este artículo pretendo pasar revista a algunos de los problemas que me parecen interesantes con respecto a la situación actual de la superconductividad. La perspectiva de que pueda existir superconductividad a temperatura ambiente, abre un interés especial tanto desde el punto de vista teórico como desde el punto de vista de las aplicaciones tecnológicas. Aunque la superconductividad convencional (ver más abajo) sido explicada en todo detalle, la superconductividad de alta temperatura crítica que bien podría ser no-convencional, permanece como una de las incógnitas más fascinantes y un reto intelectual inmenso para los investigadores del tema en los albores del Siglo XXI, como ya lo mencioné.

La definición de la superconductividad, los pares de Cooper, la teoría BCS y su extensión, las ecuaciones de Eliashberg, que explican la superconductividad convencional son temas que trataré en una primera parte. En la segunda, abordaré el tema de los nuevos superconductores de alta temperatura crítica, la superconductividad y el magnetismo, un tema de gran interés. Omitiré dos temas que merecen una amplia discusión y son de gran interés actualmente. El primero es

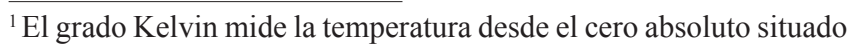
hacia -278 grados Celcius. "superconductividad, grafeno y grafito" y el segundo, el de la predicción de la temperatura crítica de los superconductores, un reto enorme que no ha sido resuelto totalmente aun. Poder predecir de primeros principios la temperatura crítica de los superconductores abriría la puerta a la ingeniería teórica de materiales superconductores con enormes consecuencias. De esto es de lo que trata esta breve presentación.

\section{Definición de superconductividad}

La superconductividad se presenta en sistemas de carácter metálico, es decir, en sistemas que tienen estados ocupados en una banda de conducción. Hay dos experimentos que definen la superconductividad en una muestra en volumen. El primero es la pérdida total de la resistencia a una cierta temperatura que es característica de cada superconductor y que recibe el nombre de temperatura crítica, Tc. En la Figura 1, se ilustra el resultado de una medida de la resistencia vs. temperatura en el momento del paso al estado superconductor. El segundo experimento es el efecto Meissner- Ochsenfeld que consiste en que una muestra expulsa el flujo magnético $\mathrm{y}$, como consecuencia, flota sobre un imán, al pasar al estado superconductor. Esta propiedad se ha utilizado para construir trenes que literalmente flotan sobre la vía y, al evitar el rozamiento, pueden alcanzar velocidades del orden de $500 \mathrm{kms} / \mathrm{h}$ sin desgastar los rieles. Para viajes del orden de $1000 \mathrm{Km}$, estas velocidades compiten con las de un avión ya que, en este último caso, los tiempos de abordaje se añaden al del viaje mismo. El Japón está a la cabeza en este tipo de aplicaciones. El experimento se ilustra en la Figura 2.

Cuando una muestra volumétrica pierde la resistencia a una Tc y, a esa misma temperatura aparece el Efecto Meissner, la reconocemos (solo entonces) como un superconductor.

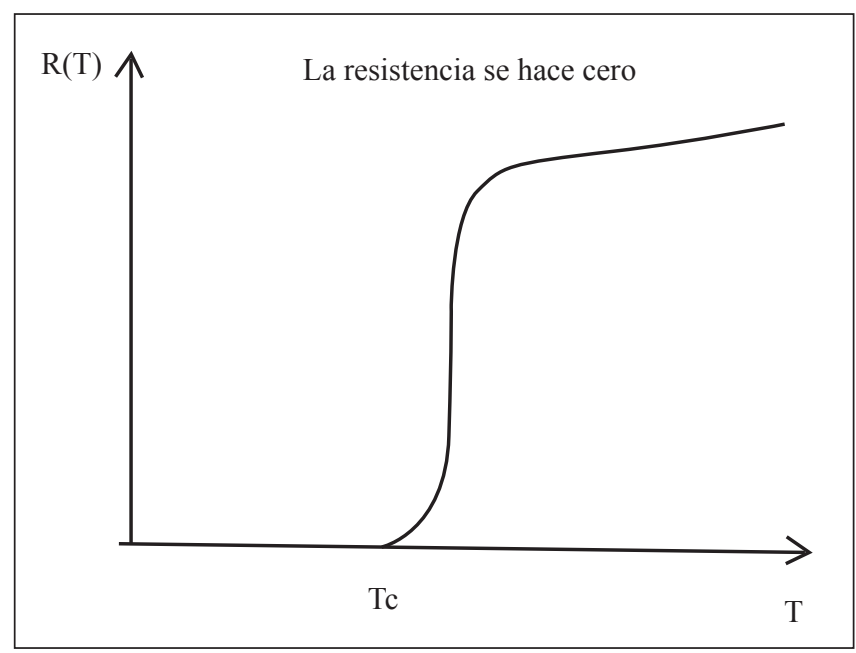

Figura 1. La resistencia de un superconductor va a cero a una cierta temperatura llamada crítica. 


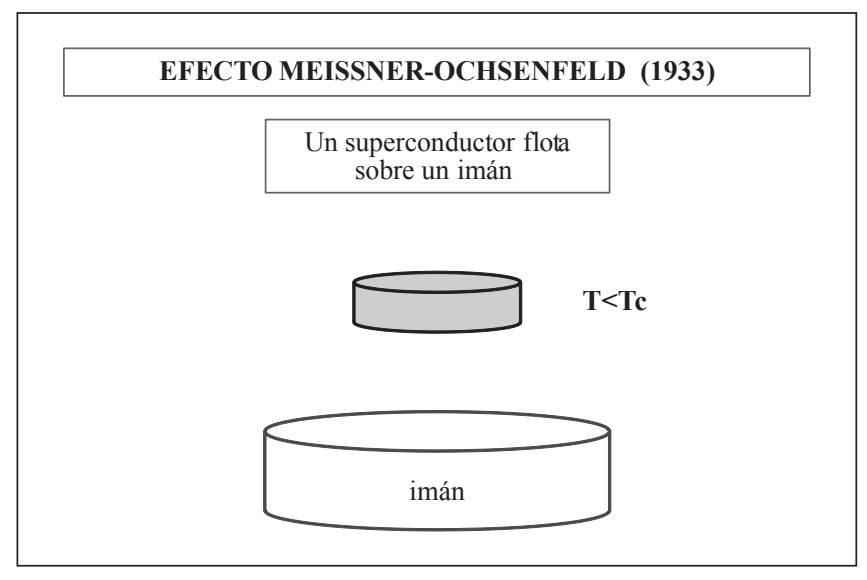

Figura 2. El Efecto Meissner-Ochsenfeld define la superconductividad junto con la pérdida total de la resistencia.

Es importante distiguir entre un conductor perfecto $\mathbf{y}$ un superconductor ya que, de acuerdo a las Ecuaciones de Maxwell, un conductor perfecto, si bien tiene resistencia cero, es un sistema termodinámicamente metaestable y, por lo tanto, no se le puede aplicar las leyes de la termodinámica y de la estadística que requieren que el sistema esté o pueda llegar a una estado de equilibrio termodinámico. Las ecuaciones de Maxwel relacionan el campo electrico y la conductividad de acuerdo a la Ley de Ohm. Un conductor perfecto tiene una conductividad infinita y es fácil demostrar que esto implica una inducción magnética siempre constante. Un superconductor la tiene siempre, en toda circunstancia, igual a cero. Se puede mostrar que este hecho implica que se trata de un sistema en equilibrio termodinámico [10]. Un superconductor expulsa el flujo magnético y constituye un diamagneto perfecto, como se ilustra en la Figura 3
B

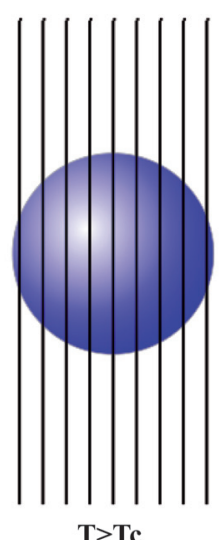

B

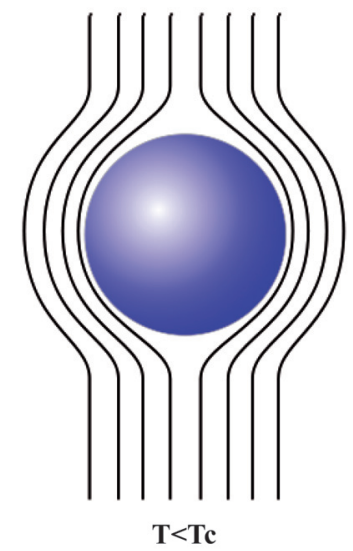

Figura 3. Un superconductor excluye el flujo magnético de su volumen y constituye un diamagnético perfecto. Ver texto.

\section{Pares de Cooper}

El concepto fundamental de la superconductividad lo constituyen los pares de Cooper. La superconductividad es la física de los pares de Cooper [11,12]. El concepto está a la base de la explicación del hecho que un superconductor pierde la resistencia pero, simultáneamente, los mejores superconductores ${ }^{2}$ son los peores conductores ${ }^{3}$. Esto quiere decir que los mejores superconductores tienen una mayor interacción con la red, lo cual parece, a primera vista, contradictorio con la idea de resistencia cero. El trabajo original de Cooper [4] de donde salió la idea de los pares de Cooper, habla de dos electrones que se atraen, colocados por encima de una superficie de Fermi (ver abajo). El Principio de Pauli (ver abajo) juega un papel fundamental para lograr este resultado ya que dos electrones libres en un espacio tridimensional sometidos a una interacción atractiva, solo pueden producir un estado ligado si su energía está por encima de un cierto umbral. Sin embargo, cuando están por encima de una superficie de Fermi, la dimensión efectiva del sistema es dos y cualquier atracción infinitesimal produce un estado ligado de los dos electrones [13]. En el caso de la superconductividad convencional, la atracción la suple la red, más exactamente, la interacción electrón-fonón, como pasamos a ver, en más detalle.

\subsection{Electrones, iones y fonones.}

Los metales se caracterizan por tener electrones libres dentro del espacio delimitado por los iones que forman la red cristalina. En la parte superior de la Figura 4, se puede apreciar un ejemplo de red cristalina. Tiene iones en los vértices de un cubo y en el centro del mismo.

Esta red cristalina se llama centrada en el cuerpo (CC). En inglés recibe el nombre de body centered cubic (bcc). En la misma figura, muestro cómo una cadena de iones está rodeada de electrones libres que son los que conducen la corriente. Gracias al hecho de que están libres, un cable de cobre puede conducir corriente sometido a un potencial producido por una pequeña pila de $1.5 \mathrm{~V}$. Pero no pueden salirse del volumen definido por los iones, es decir, por la red. Para sacarlos se necesita energía. A la energía necesaria para sacar un electrón de un metal, se le llama función de trabajo. Se saca un electrón de un metal, por ejemplo, irradiándolo con una energía mayor a su función de trabajo. A ésto se le conoce como efecto fotoeléctrico por cuya explicación, Einstein recibió el Premio Nobel en 1921. Para hacer circular una corriente por un cable se necesita un potencial (una diferencia de potencial, más correctamente) que haga circular los electrones. Pero ¿por qué se manifiesta una resistencia?

\footnotetext{
${ }^{2}$ Es decir, los que tienen una temperatura crítica más alta.

${ }^{3}$ Es decir, los que tienen una resistencia mayor.
} 


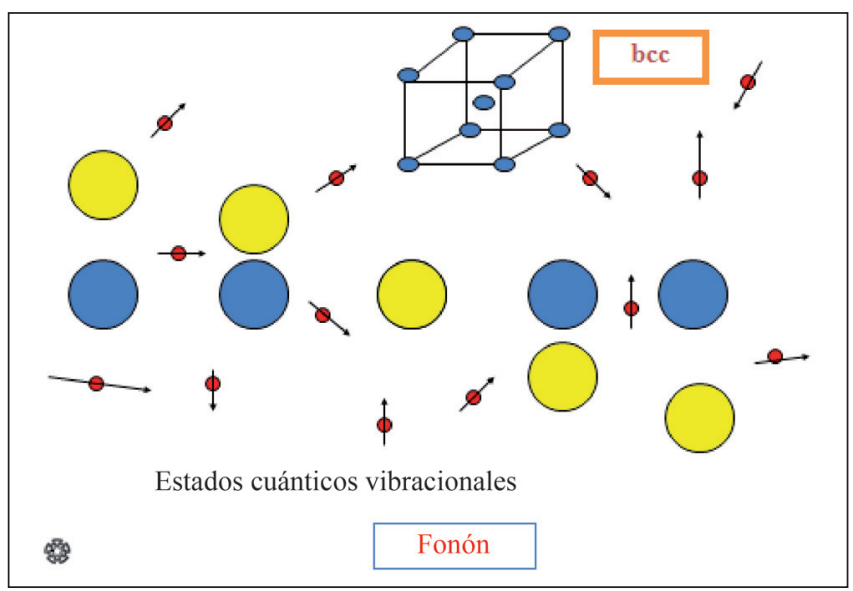

Figura 4. Un metal tiene electrones libres que son los conductores de la corriente. La resistencia que observamos se produce por interacción con la red. La red tiene estados colectivos cuánticos que se caracterizan por valores discretos de la energíay el momento, llamados estados vibracionales como se ilustra en esta figura (ver texto).

La manifestación más importante del paso de la corriente es el calor que se genera ${ }^{4}$. Este se produce por interacción entre los electrones libres y la red. Esta interacción constituye una transferencia de energía del sistema electrónico (que la adquiere del potencial externo) al sistema iónico. La energía se termaliza, subiendo la temperatura de todo el sistema que se manifiesta como calentamiento hacia el exterior. Los iones que forman la red son un sistema cuántico que tiene varios estados de energía posibles que se llaman estados vibracionales. Son estados de vibración colectiva (forman una cuerda como se ilustra en la Figura 4). Una red presenta varios estados vibracionales que se caracterizan por su energía y su momento. Las energías están cuantizadas, es decir, son niveles discretos. Cuando los electrones y los iones intercambian energía y momento, la red pasa de un estado vibracional a otro. El espectro de estados vibracionales es una característica de cada sistema específico (de un cristal). La diferencia en energía y momento entre dos estados vibracionales, constituye la única transferencia posible entre iones y electrones al interactuar entre sí. A esa diferencia se le llama fonón. Un fonón tiene energía y momento como cualquier partícula, pero no es una partícula en el sentido estricto. Por eso se le denomina cuasi-partícula. Obviamente hay varios fonones en un cristal (todas las diferencias posibles entre estados vibracionales) Los fonones son una característica específica de cada sistema cristalino.

\footnotetext{
${ }^{4}$ Nos interesa el calor cuando calentamos el café, por ejemplo. Pero si trasladamos de una hidroeléctrica a una ciudad electricidad, el calor es una pérdida que deseamos evitar (se puede llegar a perder hasta un 35\% de la energía transportada que solo sirve para calentar el aire a lo largo de la línea eléctrica). La superconductividad se usa para evitar esas pérdidas.
}

\subsection{Ondas de Bloch. Primera Zona de Brillouin.}

Los iones vibran en estados colectivos llamados estados vibracionales, como acabamos de ver. Los electrones están, unos, atados al ión pero, otros, en los metales, están libres dentro del espacio generado por la red. Los estados electrónicos están cuantizados también, pero algunos de ellos resultan estar tan cerca (en energía) unos de otros que mejor los dividimos en grupos que llamamos bandas. Entonces saber cuáles son los estados de energía de los electrones en un metal es conocer sus bandas de energía. Hablamos de la estructura electrónica de bandas. Estas se obtienen, al igual que los estados vibracionales de los iones, por medio de la solución de la ecuación de Schödinger independiente del tiempo.

$$
H \psi(r)=\varepsilon \psi(r)
$$

donde $\mathrm{H}$ es el operador Hamiltoniano del sistema, definido como la suma de $\mathrm{T}$ (operador de energía cinética) más $\mathrm{V}$ (operador de energía potencial), $\psi(\mathrm{r})$ es la función de onda y $\varepsilon$ la energía correspondiente. El volumen de un metal está compuesto por muchas redes cristalinas como la descrita arriba. En la teoría, suponemos que las redes cubren todo el espacio. Esta hipótesis lo que implica, en realidad, es separar la descripción de un volumen del de una superficie. Pero la hipótesis de un volumen infinito y compuesto por unidades iguales (la red cristalina) requiere una descripción tal que la función de onda sea periódica. Esta condición se introduce explícitamente por medio de las llamadas ondas de Bloch.

$$
\psi_{\mathbf{k}}(r)=e^{i \mathbf{k} \cdot \mathbf{r}} u_{\mathbf{k}}(r)
$$

donde $\mathrm{u}(\mathbf{r}+\mathbf{R})=\mathrm{u}(\mathbf{r})$. $\mathbf{R}$ es tal que $\mathrm{u}(\mathbf{r})$ tiene la periodicidad de la red. Entonces, describimos un electrón dentro de un cristal metálico por medio de una función que es el producto de una onda plana y de otra función que tiene la periodicidad de la red.

El número cuántico que caracteriza las diferentes soluciones, el vector k, no es cualquiera, describe los diferentes estados electrónicos posibles. Es importante suprimir el doble conteo. Los k's diferentes se encuentran dentro de un cierto espacio tridimensional llamado Primera Zona de Brillouin que es parte del espacio recíproco, el espacio-k. Puede mostrase que la relación entre el momento del electrón, $\mathbf{p}, \mathrm{y}$ el vector $\mathbf{k}$ es

$$
\mathbf{p}=\hbar \mathbf{k}
$$

donde $\hbar$ es la constante característica de los fenómenos cuánticos, la constante de Planck. Los electrones dentro de un cristal tienen una compleja estructura de bandas. Sin embargo, para fines de definir con claridad el concepto de superficie de Fermi, podemos tomar la energía total de cada estado igual a la energía total de una partícula libre,

$$
\varepsilon_{\mathbf{k}}=\frac{\mathbf{p}^{2}}{2 m}=\frac{\hbar^{2} \mathbf{k}^{2}}{2 m}
$$


En este caso, los diferentes estados de energía dependen solo del valor absoluto del vector de onda $\mathbf{k}$ ( $\hbar k)$ es el momento cristalino, que caracteriza cada estado electrónico). Si dibujamos los diferentes valores de la energía (que no depende de la dirección), en una estructura tridimensional, obtenemos una esfera en el espacio de las k's como se ilustra en la siguiente Figura 5.

\subsection{El Principio de Pauli. La Superficie de Fermi.}

¿Por qué los estados electrónicos forman una esfera? ¿Por qué no pueden quedarse todos en el estado de mínima energía? La razón es el Principio de Pauli que dice que no puede haber dos fermiones (electrones) ocupando el mismo estado. Ya he mencionado la importancia de este principio en la formación de los Pares de Cooper [13]. Cada estado ocupa un cierto espacio finito del espacio fase. El espacio fase es la combinación del espacio tridimensional real y del espacio de los vectores k. Por esa razón, a medida que colocamos los electrones en los estados del espacio $\mathbf{k}$ tenemos que ir subiendo en energía porque el espacio disponible entre $\mathbf{k}$ y $\mathbf{k}+\mathbf{d k}$ se va agotando a medida que lo cubrimos con electrones. Es así como formamos el mar de Fermi y, una vez que hemos colocado todos los electrones, se forma la superficie de Fermi (una esfera, en este caso) que separa la energía de los estados ocupados de los desocupados. Es la misma idea que ocupar una escalera muy larga con un número de personas

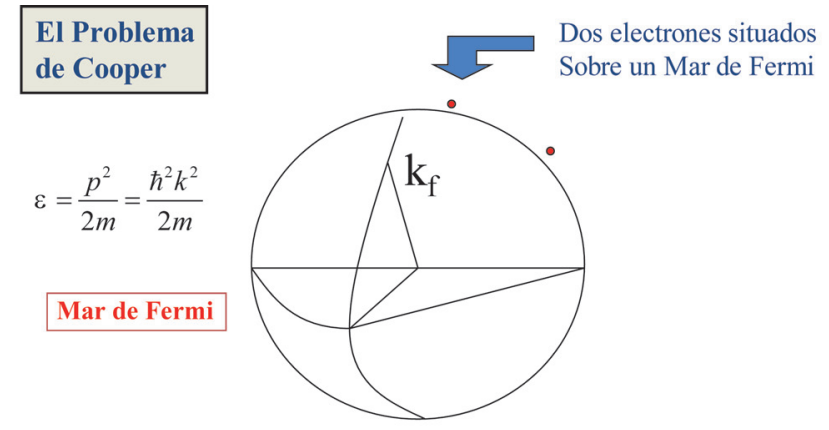

Si existe alguna atracción entre los dos electrones, el sistema sufre una transición de fase a $\mathrm{T}=\mathrm{Tc}$ que involucra a un cierto número de electrones cercanos al nivel de Fermi. Al salir estos electrones del espacio de Hilbert de los electrones "libres", se produce una contracción en la esfera de Fermi

Figura 5. Los estados de energía de los electrones ocupados dentro de un metal forman, en tres dimensiones, una esfera llamada Mar de Fermi cuya superficie se conoce como la Superficie de Fermi. En el caso real la superficie difiere de la de una esfera porque la energía no es exactamente la de una partícula libre. El vector $\mathbf{k}$ de mayor valor absoluto recibe el nombre de vector de Fermi y define la máxima energía que tienen los electrones dentro del metal a temperatura $0 \mathrm{~K}$. En este caso, se trata de un estado altamente degenerado ya que todas las direcciones que terminan sobre la superficie representan estados con la misma energía. La figura explica también como se forma un Par de Cooper (ver texto). menor al número de peldaños cuando solo cabe una persona en cada peldaño, sin dejar ningún peldaño vacío. Va a haber un peldaño más allá del cual ya no hay personas. Notemos una cosa. Si pedimos que suban un peldaño, solo el de más arriba puede hacerlo ya que es el único que tiene vacío el peldaño inmediato superior. Igualmente, los electrones de la superficie de Fermi son los más activos, puesto que son los que pueden recibir energía, ya que tienen estados vacíos a donde dirigirse al recibir dicha energía y al igual que en el caso de la escalera, solo puede haber uno en cada estado. La superficie de Fermi tiene una gran importancia en la física de los metales.

\subsection{Los Pares de Cooper.}

La superconductividad es la física de los pares de Cooper. Este concepto es, por lo tanto, la esencia de la superconductividad. Volvamos a la Figura 5. Tenemos dos electrones colocados por encima de un mar de Fermi. Es decir, no pueden perder (ceder) energía porque no hay estados de menor energía que se puedan ocupar. Si, por alguna causa, se produce una atracción entre los dos electrones, entonces se produce una transición de fase en la cual los electrones forman un estado ligado. La energía de amarre de este par es negativa y el nuevo estado se convierte, entonces, en el estado base. A estos pares se les denomina pares de Cooper. ¿Qué sucede entonces, en el estado superconductor?

\section{La teoría BCS}

Desde el descubrimiento de la superconductividad en 1911, el conocimiento científico desarrolló conceptos nuevos que permitieron encontrar una explicación teórica de la superconductividad. La ecuación de Schrödinger se estableció en 1927 [14], la interpretación de la misma es, incluso hoy, motivo de discusión. La interpretación hecha por Bohr [15] a pesar de las objeciones de Einstein [16] se impuso y las aplicaciones tecnológicas de la mecánica cuántica revolucionaron el siglo XX. La existencia de la superficie de Fermi en los metales fue establecida hacia los años cincuentas, como ya lo mencioné. La superconductividad es un fenómeno cuántico no-relativista macroscópico de muchos cuerpos. La teoría de campo fue establecida durante la primera mitad del siglo XX. La versión no-relativista explica de una manera muy elegante, partiendo de un Hamiltoniano gran canónico (funciones de Green dependientes de la temperatura), el fenómeno de la superconductividad [17]. Fue en el año de 1957, cuando, uniendo todos estos conocimientos, apareció la primera teoría de este fenómeno [5], a más de 45 años de descubierta. Entonces, en sus primeros cincuenta años, esencialmente, la investigación de la superconductividad, como resultado más sobresaliente, logró establecer una teoría del fenómeno aceptada en forma general. Fue formulada por John Bardeen, Leo Cooper y Alexander Schrieffer y lleva el 
nombre de teoría BCS. Pero fue solo hasta comienzos de los setentas cuando se reconoció la validez y la importancia de esta teoría y se les concedió el Premio Nobel en 1972.

Consiste la teoría BCS en la hipótesis acertada de que la superconductividad se forma porque, al bajar la temperatura hasta un valor crítico como se muestra en la Figura 1, el sistema forma pares de Cooper con los electrones cercanos al nivel de Fermi.

El punto de partida es un Hamiltoniano que describe la existencia de electrones y de fonones:

$$
H_{0}=\sum_{\mathbf{k}} \varepsilon_{\mathbf{k}} c_{\mathbf{k}}^{+} c_{\mathbf{k}}+\sum_{\mathbf{q}} \hbar \omega_{\mathbf{q}} a_{\mathbf{q}}^{+} a_{\mathbf{q}}
$$

donde los operadores $\mathrm{C}_{\mathrm{k}}^{+} \mathrm{y} \mathrm{C}_{\mathrm{k}}$ son operadores de creación y aniquilación de electrones, respectivamente, y $\operatorname{los}{a^{+}}_{\mathrm{q}} \mathrm{y} \quad a_{\mathrm{q}}$ son los correspondientes para fonones. Los operadores son operaciones matemáticas que se hacen sobre la función de onda que describe el sistema. El producto de un operador de aniquilación y otro de creación del mismo carácter constituye un contador ya que el primero quita una partícula al sistema con el resultado de que si ésta existe, se obtiene un sistema del mismo tipo con una partícula menos (si no existe la partícula, no la puede quitar y el resultado es cero); el otro operador restituye la partícula dejando el sistema idéntico. El número de veces que puedo realizar la operación me dice cuantas partículas hay, ya que los términos correspondientes a las que no existen, contribuyen con cero $[11,17] ; \hbar \omega_{\mathrm{q}}$ es la energía del fonón con momento $\mathbf{q}$ y $\varepsilon_{\mathrm{k}}$ es la energía del electrón con vector de onda $\mathbf{k}$. La ecuación contabiliza, entonces, cuanta energía hay tanto en el sistema electrónico, como en el fonónico. El hamiltoniano describe la existencia de los elementos fundamentales en un metal: electrones y fonones. No hay un término que permita la interacción, es decir, que permita que pase energía de un sistema al otro. Vamos a agregarlo ahora, vamos a agregar la interacción electrónfonón. El término que tenemos que agregar debe decir: un electrón absorbe (cede) la energía de un fonón. Entonces, un electrón en un estado $\mathbf{k}$ pasa a otro estado $\mathbf{k}^{\prime} \mathbf{y}$, al mismo tiempo, destruye (crea) ${ }^{5}$ un fonón con energía y momento igual a la diferencia entre los dos estados electrónicos. Esta frase se escribe asi,

$$
H_{I}=\sum_{\mathbf{k}, \mathbf{k}^{\prime}, \mathbf{q}} M_{\mathbf{k}, \mathbf{k}^{\prime}}\left(a_{-\mathbf{q}}^{+}+a_{\mathbf{q}}\right) c_{\mathbf{k}}^{+} c_{\mathbf{k}^{\prime}}
$$

El primer operador destruye un electrón en el estado k', el segundo crea un electrón en el estado k. Hay dos opciones para conservar energía y momento. Una, crear un fonón (primer término entre paréntesis) y, entonces el balance de los momentos (y de la energía) es:

\footnotetext{
${ }^{5}$ Cuando la red pasa de un estado vibracional a otro de mayor energía decimos que se crea un fonón. Si es de menor energía decimos que se aniquila un fonón.
}

teníamos k', generamos k y -q. Por lo tanto la suma de los dos últimos debe ser igual al primero: $\mathbf{k}^{\prime}=\mathbf{k}-\mathbf{q}$. En la segunda opción (segundo término en la suma), tenemos k', generamos $\mathbf{k} \mathbf{y}$ perdemos (destruimos) q. Por lo tanto $\mathbf{k}^{\prime}=\mathbf{k}-\mathbf{q}$. Y vemos que la idea de definir el momento del operador de creación "-q" es para que la ecuación de conservación de momento tenga la misma forma en ambos casos. $\mathrm{M}_{\mathrm{kk}}$, es un término que mide, esencialmente, la probabilidad de que cada uno de esos eventos ocurra. La suma es por todos los eventos posibles. El hamiltoniano de Fröhlich [3] que describe un sistema de electrones y de fonones interactuando entre sí, queda entonces, así:

$$
H=H_{0}+H_{I}
$$

Queremos describir, dentro de este formalismo, a los pares de Cooper.

Repito, la superconductividad es la física de los pares de Cooper, es decir, que es un sistema donde solo existen pares de Cooper (aparte de la red) en el mismo sentido que un metal en estado normal es un sistema donde solo existen electrones libres (aparte de la red).

Un par de Cooper está formado por dos electrones que se atraen. ¿Quién provee la atracción?. En la Figura 6 se esquematiza la atracción entre dos electrones mediada por la red. El electrón 1 polariza la red. Esto genera una zona de exceso de carga positiva que resulta en una atracción para otro electrón 2. Entonces, la polarización dinámica generada por el electrón 1, es seguida por el electrón 2, lo cual constituye una atracción efectiva. Este hecho puede caricaturizarse asi: si dos chicos desean andar con la misma chica, lo más probable es que donde esté la chica quieran estar los dos, así se odien, en realidad. La chica constituye una atracción efectiva entre los dos chicos. Como podemos ver en la Figura 6, el electrón 1 da energía a la red (la polariza, crea un fonón) y el 2 la toma

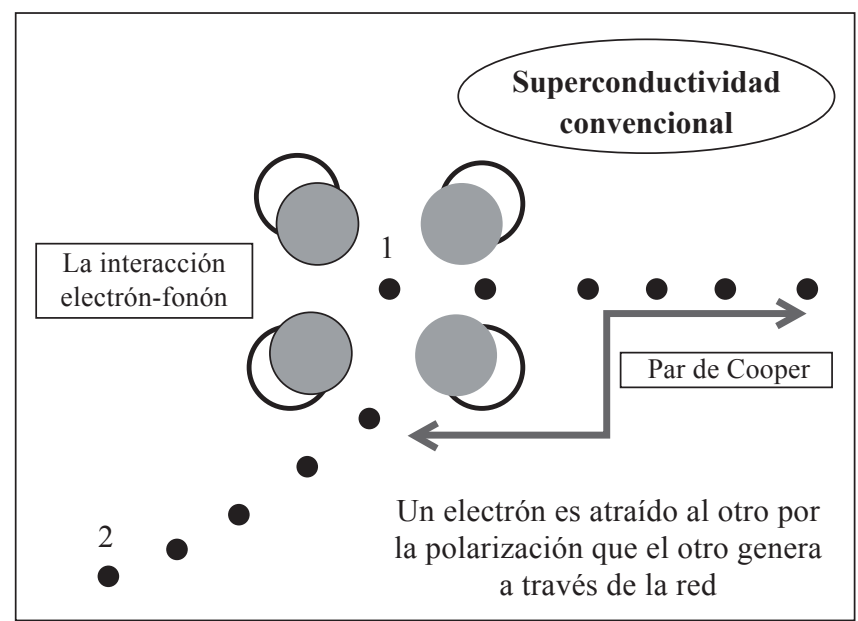

Figura 6. La atracción entre dos electrones en un medio cristalino. 
(se acelera en el campo causado por la polarización, destruye el fonón creado por el electrón 1). Esto lo podemos dibujar por medio de un diagrama de Feynman [17] que son muy ilustrativos $^{6}$. En la Figura 7, el diagrama muestra como un electrón con momento -k, energía $\varepsilon_{-\mathbf{k}}$ y espín hacia abajo ${ }^{7}$ crea un fonón con energía $\hbar \omega_{q}$ y momento q que otro electrón con momento $\mathbf{k}$, energía $\varepsilon_{\mathbf{k}}$ y espín hacia arriba absorbe (destruye) de acuerdo al proceso descrito en la Figura 6. La red no se queda, al final, ni con energía, ni con momento. Nótese que ésto explica por qué los mejores superconductores son los peores conductores, como mencioné más arriba. La razón es que si la interacción con la red es grande (mal conductor), la energía de polarización lo será también y, por lo tanto, la energía necesaria para romper esa atracción de la red, será mayor lo cual implica que la temperatura a la cual se romperá la correlación (que es representativa de esa energía) será también mayor (un mejor superconductor). La interacción puede, entonces, se puede describir como dos electrones que intercambian un fonón. $\mathrm{Y}$ el proceso debe repetirse de forma continua para que siempre haya solo pares de Cooper. Es decir, la dispersión lleva de un par de Cooper a otro. Nótese que este proceso se respeta en el que se ilustra en la Figura 7. Un par de Cooper se dispersa en otro par de Cooper.

\section{La superconductividad es la física de los Pares de Cooper}

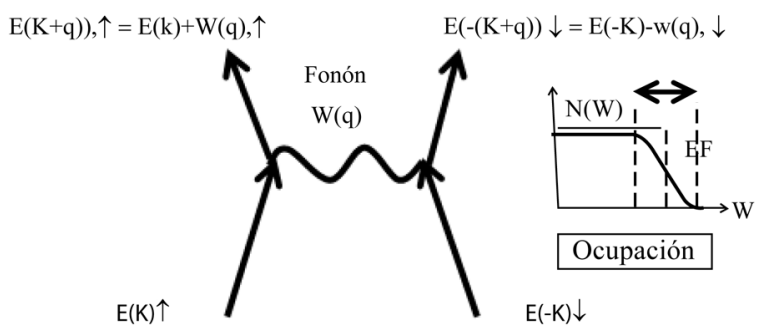

La condición es que la dispersión lleve de un Par de Cooper a otro Par de Cooper intercambiando un fonón, sin dejar ni energía, ni momento, en la red.

Figura 7. El Par de Cooper

\footnotetext{
${ }^{6}$ Un diagrama de Feyman remplaza expresiones matemáticas de la teoría cuántica de campos que describen la interacción entre partículas por diagramas que permiten visualizar muy claramente la física que hay detrás.

${ }^{7}$ El espín es un número cuántico relativista. En mecánica cuántica no relativista, se agrega para contar los estados en forma conveniente ya que dos estados electrónico con la misma energía y el mismo momento pero diferente espín, son diferentes y pueden ser ocupados por un electrón cada uno. El electrón tiene espín $1 / 2$ con dos proyecciones (estados diferentes) : $-1 / 2$ y $+1 / 2$ que también se suelen representar con flechas indicando espín hacia "arriba" y hacia "abajo". Esta designación tiene que ver con experimentos en presencia de campos magnéticos. Los fermiones tienen espín semi-entero.
}

¿Qué implica esto? La implicación física de este hecho puede leerse de la Figura 7. Si originalmente, ambos electrones tenían su energía sobre la superficie de Fermi o muy cercana a éste, al intercambiar un fonón, uno de ellos va hacia un estado situado por debajo de esa energía y el otro a un estado por encima. ¿Qué tan abajo y qué tan arriba? Eso depende del tamaño de la energía del fonón. En esto influye la ocupación. A $\mathrm{T}=0 \mathrm{~K}$, un metal en estado normal tiene sus electrones libres en estados por debajo del nivel de Fermi. No hay ninguno por encima (como en el caso de la escalera). Esto es, la probabilidad de ocupación, $\mathrm{N}(\varepsilon)=1$, si $\varepsilon<\varepsilon_{\mathrm{F}}$ y $\mathrm{N}(\varepsilon)=$ 0 , si $\varepsilon>\varepsilon_{\mathrm{F}}$. Solo si la temperatura, $\mathrm{T}$, es mayor que cero, $\mathrm{T}>$ 0 , habrá electrones ocupando estados por encima de $\varepsilon_{\mathrm{F}}$. Pero la dispersión de par de Cooper a par de Cooper, que se necesita para mantener el estado superconductor, implica que, a $\mathrm{T}=0 \mathrm{~K}$ (ver Figura 7), haya estados disponibles por debajo del nivel de Fermi y que, por encima del nivel de Fermi algunos estados estén ocupados. Solo así la dispersión descrita en la Figura 7 puede realizarse. La probabilidad de ocupación va a ser diferente, va a ser como la que se muestra en la Figura 7. Como puede verse, el ancho de la zona energética donde solo existen pares de Cooper, va a determinar que tan grande es la energía de amarre de los pares. Este ancho está determinado por la máxima energía que los electrones pueden intercambiar en un material específico y es, por lo tanto, una característica de cada superconductor. En esta región energética no existen electrones libres. Por debajo de ella sí. Por lo tanto, si queremos excitar un electrón libre en un superconductor, debemos dar la suficiente energía para saltar la barrera energética. Esa barrera que, como vemos en la Figura 7, se sitúa, simétricamente, por encima y por debajo del nivel de Fermi, recibe el nombre de brecha del estado superconductor, $2 \Delta$. Es simétrica alrededor del nivel de Fermi, porque un electrón puede absorber el fonón de máxima energía existente en el material y se dispersa hacia un estado de mayor energía o lo puede ceder (pierde esa energía). Esperamos, obviamente, que la brecha esté directamente relacionada con la temperatura crítica. Una de las ecuaciones más importantes de una teoría de la superconductividad es, en consecuencia, la que permite calcular la brecha.

\subsection{Las Ecuaciones BCS}

El hamiltoniano de Frölich (electrón-fonón) se puede escribir (después de haber hecho una transformación canónica) de la siguiente manera, más apropiada para describir los pares de Cooper.

$$
H^{\prime}=H_{0}+\sum_{\substack{\mathbf{k}, \mathbf{k}^{\prime} \\ \mathbf{k}-\mathbf{k}^{\prime}=\mathbf{q}}}\left|M_{\mathbf{q}}\right|^{2} \frac{\hbar \omega_{\mathbf{q}}}{\left(\varepsilon_{\mathbf{k}}-\varepsilon_{\mathbf{k}-\mathbf{q}}\right)^{2}-\left(\hbar \omega_{\mathbf{q}}\right)^{2}} c_{\mathbf{k}^{\prime}+\mathbf{q}}^{+} c_{\mathbf{k}-\mathbf{q}}^{+} c_{\mathbf{k}} c_{\mathbf{k}}
$$

donde todos los términos ya están definidos y, estrictamente hablando, hemos omitido términos que no contribuyen en el estado superconductor. Observemos que, si las energías electrónicas en el denominador se encuentran muy cercanas 
entre sí, su diferencia puede ser menor que la del fonón y la interacción se vuelve atractiva (negativa). Los operadores de creación y aniquilación de los electrones, describen un par de Cooper yendo a otro par de Cooper cuando $\mathbf{k}=-\mathbf{k}$ '. El Hamiltoniano conserva el momento.

Toda esa física fue muy elegantemente simplificada en la teoría BCS [5] describiendo el sistema de la siguiente manera,

$$
H_{B C S}=\sum_{\mathbf{k}} \varepsilon_{\mathbf{k}}\left(c_{\mathbf{k}}^{+} c_{\mathbf{k}}+c_{-\mathbf{k}}^{+} c_{-\mathbf{k}}\right)-\sum_{\mathbf{k}, \mathbf{k}^{\prime}} V_{\mathbf{k} \mathbf{k}^{\prime}} c_{\mathbf{k}^{\prime}}^{+} c_{-\mathbf{k}^{\prime}}^{+} c_{-\mathbf{k}} c_{\mathbf{k}}
$$

Observemos que el hamiltoniano BCS describe exactamente la dispersión de un par de Cooper a otro par de Cooper y que la condición que vimos arriba, $\mathbf{k}^{\prime}=\mathbf{- k}$, ha sido incluida explícitamente. Una simplificación adicional fue definir la atracción como una constante que solo actúa en el rango de energía de los fonones, es decir, en el intervalo de la brecha, esencialmente,

$$
\begin{aligned}
& V_{\mathbf{k k}^{\prime}}=V=\text { cte, si } \varepsilon_{\mathrm{F}}-\hbar \omega_{\max }<\varepsilon<\varepsilon_{\mathrm{F}}+\hbar \omega_{\max } \\
& V_{\mathbf{k k}^{\prime}}=0 \text { en cualquier otro intervalo }
\end{aligned}
$$

Es decir, que la atracción se ejerce solo cuando el fonón que se intercambia existe. La frecuencia, $\hbar \omega_{\max }$, es la del fonón de máxima energía que se suele identificar con la frecuencia de Debye del material. Ante la imagen física que hemos construido, es claro que identificar esta máxima frecuencia con la frecuencia de Debye [18], es discutible. Esta aproximación para el potencial le quita la dependencia en $\mathbf{k}$ también a la brecha. Convirtiendo las sumas en integrales y suponiendo constante la densidad de estados e igual a su valor sobre el nivel de Fermi, un algebra directa nos lleva a la ecuación para la brecha $(\mathrm{a} \mathrm{T}=0 \mathrm{~K})[12,19]$.

$$
\Delta_{0}=\frac{\hbar \omega_{\max }}{\sinh \left(\frac{1}{N\left(\varepsilon_{F}\right) V}\right)} \approx 2 \hbar \omega_{\max } e^{\frac{-1}{N\left(\varepsilon_{F}\right) V}}
$$

La ecuación para la temperatura crítica, Tc, se deriva en forma directa,

$$
T_{c}=1.14 \hbar \omega_{\max } e^{\frac{-1}{N\left(\varepsilon_{F}\right) V}}
$$

de donde se deduce una de las relaciones universales por las cuales es famosa la teoría BCS,

$$
\frac{2 \Delta_{0}}{K_{B} T_{c}}=3.52
$$

que es, precisamente, la relación entre la Tc y la brecha mencionada arriba. Las teoría BCS es cualitativa ya que la atracción, el parámetro $\mathrm{V}$, en un sistema real depende tanto de la frecuencia del fonón, $\omega$, como del vector de onda del electrón k. Además, el parámetro V no puede determinarse con exactitud, ni teórica, ni experimentalmente. Sin embargo, las predicciones cualitativas de la BCS, resultaron tan correctas y convincentes que no quedó duda de que la superconductividad es la física de los pares de Cooper. Este paso fundamental, abrió el camino para desarrollar la misma idea con las técnicas de teoría de campo norelativista (teoría de muchos cuerpos). El resultado son las Ecuaciones de Eliashberg que dan plena cuenta de todos los superconductores convencionales ${ }^{8}$.

En el estado normal, la densidad de estados en función de la temperatura, para un gas de electrones libres, va como la raiz cuadrada de la energía. En el estado superconductor la expresión BCS es

$$
N_{s}=N\left(\varepsilon_{F}\right) \frac{\varepsilon-\varepsilon_{F}}{\sqrt{\left(\varepsilon-\varepsilon_{F}\right)^{2}-\Delta^{2}}}
$$

Como es obvio, el paso al estado normal al subir la temperatura implica que $\Delta$ es, en general una función de la temperatura, $\Delta(\mathrm{T})$. El denominador de la Ec. 14, da la energía de las exitaciones elementales en teoría BCS.

Propiedades muy conocidas del estado superconductor predichas por BCS, son el salto en el calor específico y el coeficiente de atenuación ultrasónica. La superconductividad es una transición de fase de segundo orden en ausencia de campo magnético lo cual implica que el calor específico tiene una discontinuidad en $\mathrm{T}=\mathrm{Tc}$. La atenuación ultrasónica atestigua que, en un superconductor, el sonido no se atenúa en absoluto a $\mathrm{T}=0 \mathrm{~K}$, hecho que se ha podido comprobar experimentalmente ${ }^{9}$. Otra relación universal involucra el salto en el calor específico electrónico, en unidades del mimo a $\mathrm{T}=\mathrm{Tc}^{+}$, justo arriba de la transición, en el estado normal. Ver detalles en $[10,19]$, por ejemplo.

$$
\frac{\Delta C_{e}}{\gamma T_{c}}=1.43
$$

En conclusión, la teoría BCS formulada en 1957 [5] (Bardeen, Cooper y Schrieffer obtuvieron el Premio Nobel por ella en 1971) es el paso conceptual más importante en la explicación de la superconductividad. Demuestra que la superconductividad es la física de los pares de Cooper, es decir, el resultado de la atracción que un grupo de electrones con energía cercana o igual a la del nivel de Fermi, resiente debido a su interacción con la red (fonones). El hamiltoniano BCS simplifica la complicadísima atracción de muchos cuerpos que depende tanto de la energía de los fonones como del estado electrónico involucrado, por una constante que describe un potencial atractivo en su mínima esencia. Con ese hamiltoniano simplificado para describir un sistema de pares

\footnotetext{
${ }^{8}$ El grupo de superconductores donde los electrones forman Pares de Cooper intercambiando un fonón, se suele llamar "convencional".

${ }^{9}$ Cuando uno grita, a una distancia grande, ya no lo oyen. El sonido se atenúa. Dentro de un superconductor no.
} 
de Cooper, BCS reproduce cualitativamente, resultados como el salto en el calor específico, la variación de la brecha con la temperatura, la atenuación ultrasónica, el campo magnético crítico, entre otros muchos, y, ésto es muy importante, ofrece una explicación directa y simple del efecto isótopo. Obtiene una ecuación para la brecha en función de la temperatura y otra para la temperatura crítica. Finalmente, las relaciones universales son un resultado muy importante y que, aun hoy en dia, son un punto de referencia. Con base a este fantástico trabajo, se pudo desarrollar la misma idea de una manera más exacta, las ecuaciones de Eliashberg, que dan cuenta, en detalle, de la superconductividad convencional.

\section{Las ecuaciones de Eliashberg}

Como lo mencioné en la sección anterior, las Ecuaciones de Eliashberg [20] resultan del desarrollo en teoría de muchos cuerpos (teoría cuántica de campos no-relativista) de la física de un sistema de pares de Cooper. Para obtener resultados exactos, es necesario introducir datos detallados del sistema descrito. Estos datos incluyen, la superficie real de Fermi alrededor de la cual están los estados involucrados en la transición, la interacción electrón-fonón y los fonones existentes en el material. Toda esa información se condensa en una función, la función de Eliashberg, $\alpha^{2} F(\omega)$, que representa el espectro efectivo de la interacción electrónfonón en el estado superconductor. Esta función es el dato de entrada. Puede obtenerse tanto teóricamente como experimentalmente. Para el cálculo numérico [21] es mejor escribir las ecuaciones de Eliashberg en el eje imaginario de las energías. Las voy a escribir a continuación en función de la frecuencia (la dependencia en $\mathbf{k}$ está integrada para simplificarlas). Las escribo para que el lector tenga una idea de su complejidad. Obviamente se trata de una ecuación para la brecha. Durante la década de los setentas se presentaron varios códigos para resolverlas. El grupo de J.P. Carbotte [21] fue uno de los primeros en establecer un algoritmo adecuado para resolverlas. La ecuación puede linearizarse a $\mathrm{T}=\mathrm{Tc}$ y es más fácil de resolver. Con base en esa solución se puede implementar la solución para temperaturas inferiores y calcular las funciones termodinámicas en función de la temperatura, La ecuación [20] es

$$
\begin{gathered}
\Delta(\omega)=\frac{1}{Z(\omega)} \int_{0}^{\omega_{c}} d \omega^{\prime} \operatorname{Re}\left\{\frac{\Delta\left(\omega^{\prime}\right)}{\sqrt{\omega^{\prime 2}-\Delta^{2}\left(\omega^{\prime}\right)}}\right\}\left\{K_{+}\left(\omega, \omega^{\prime}\right)-\mu^{*}\right\} \\
(1-z(\omega)) \omega=\int_{0}^{\omega_{c}} d \omega^{\prime} \operatorname{Re}\left\{\frac{\Delta\left(\omega^{\prime}\right)}{\sqrt{\omega^{\prime 2}-\Delta^{2}\left(\omega^{\prime}\right)}}\right\}\left\{K_{-}\left(\omega, \omega^{\prime}\right)\right\}
\end{gathered}
$$

donde

$$
K_{ \pm}\left(\omega, \omega^{\prime}\right)=\int_{0}^{\infty} d v \alpha^{2} F(\omega)\left\{\frac{1}{\omega^{\prime}+\omega+v+i \varepsilon} \frac{1}{\omega^{\prime}+\omega+v-i \varepsilon}\right\}
$$

La frecuencia $\omega_{c}$ es una frecuencia de corte necesaria para la solución numérica. Hay formas de escogerla de tal manera que no influya en los resultados. La ecuación, como ya mencioné, se puede linearizar a $\mathrm{T}=\mathrm{T}$. El problema está en que el parámetro $\mu^{*}$, que es un seudopotencial que describe la repulsión electrón-electrón promediada por todas sus variables para convertirlo en una constante, no se puede obtener con la precisión suficiente ni teórica, ni experimentalmente. Por esa razón, la ecuación linealizada válida a Tc, no se usa para predecirla sino, más bien, introduciendo el valor experimental, se obtiene el valor consistente de $\mu^{*}$ por medio de la Ecuación linearizada de Eliashberg. Ese valor se incrusta en la ecuación no-lineal válida para $\mathrm{T}<\mathrm{Tc}$. El valor típico oscila entre 0.10.13 . La precisión necesaria para que el cálculo numérico no diverja es de seis decimales significativos.

De la solución se puede obtener toda la termodinámica del estado superconductor para todos los materiales convencionales. Es importante mencionar que estas ecuaciones permiten evaluar e interpretar, desviaciones de los valores de las relaciones universales BCS. Otra extensión de la BCS que es importante mencionar es la teoría de Abrikosov-Gorkov para aleaciones superconductoras [22]. Omito los detalles por razones de espacio pero es muy importante mencionarla. Una conclusión por destacar es que ni BCS, ni las Ecuaciones de Eliashberg, pueden predecir la temperatura crítica de los superconductores de primeros principios. Ese es un tema abierto. Su solución daría un tremendo empuje a la ingeniería teórica de materiales superconductores.

\subsection{La derivada funcional}

Uno de los resultados interesantes que emanan de las ecuaciones de Eliashberg es la derivada funcional de la Tc con respecto a la función de Eliashberg [23].

$$
\frac{\delta T_{c}}{\delta \alpha^{2} F(\omega)}
$$

Esta derivada nos permite saber cuál será la influencia que un cambio en la función de Eliashberg (dopando o sacando el material de la estequiometria) tiene en el valor de la $\mathrm{Tc}$ (subiéndola o bajándola). El resultado más interesante que emana de esta derivada funcional es el hecho de que existe una frecuencia privilegiada, la frecuencia óptima, $\omega_{\text {opm }}$, para cada material que determina la Tc. Esto quiere decir que los electrones se acoplan, de manera ideal para el sistema considerado, a un cierto fonón bien definido. Cuando la frecuencia no coincide exactamente con la de un fonón del material, entonces es posible que exista una forma de doparlo con éxito para subir su Tc. Cuando coinciden, el material está ya optimizado [23]. Pero lo que más llama la atención es el hecho de que, en unidades de Tc, el máximo resulta universal. Esta relación no ha sido explorada lo suficiente hacia la predicción de la temperatura crítica que, como ya mencioné, es un problema abierto. La relación universal es, 


$$
\frac{\hbar \omega_{o p t}}{K_{B} T_{c}}=\text { const }
$$

donde la constante tiene un valor entre 7 y 8 que es universal.

\section{La Teoría de Ginzburg-Landau.}

Muy importante es la teoría de Ginzburg-Landau [24] que ha sido muy fructífera para el tratamiento teórico de la superconductividad. La teoría trata de manera general, las transiciones de fase y se aplica muy bien a la superconductividad. Puede mostrarse que coincide con la $\mathrm{BCS}$ en el régimen que corresponde (cerca de Tc). Los superconductores de tipo II (casi todos los de alta Tc lo son) ${ }^{10}$ fueron descubiertos teóricamente por Abrikosov [25] en los años cincuentas como una aplicación de la Teoría de Ginzburg-Landau. Fue solo después de descubierta la superconductividad de alta Tc que se notó la importancia de ese trabajo. Abrikosov y Gorkov fueron galardonados con el Premio Nobel en el 2003. Esta teoría se encuentra en casi todos los libros de superconductividad (ver $[12,19]$ por ejemplo).

\section{Los superconductores de alta TC.}

La nueva superconductividad y algunos de los nuevos problemas. En 1986, Bednorz y Müller [8] abrieron un campo nuevo, totalmente nuevo. El de la superconductividad de alta temperatura crítica. Y digo nuevo, no solo porque las temperaturas críticas se incrementaron enormemente ${ }^{11}$ sino también porque el apareamiento entre los electrones bien podría no ser mediado por los fonones de la red. En ese sentido se habla de superconductividad no-convencional. Se ha ofrecido varias posibilidades para explicarlo pero aun hoy (noviembre 2014) no hay consenso sobre el mecanismo que da lugar a esas temperaturas críticas altas. Voy a describir algunos de los materiales superconductores que han generado un gran interés. Destacaré fundamentalmente el YBCO por ser el sistema más estudiado, quizás, y el $\mathrm{MgB}_{2}$ por ser el superconductor electrón-fonón con una temperatura mucho mayor a las conocidas entre los convencionales antes de 1986. Más adelante abordaré el tema de superconductividad y magnetismo. No trataré el tema de superconductividad en el grafeno y en el grafito por razones de espacio, muy a mi pesar porque es un campo muy activo en la investigación actual.

\footnotetext{
${ }^{10}$ Los superconductores de tipo I se distinguen de los de tipo II por la forma que adoptan cerca de la transición. Los de tipo II forman vórtices y la física de éstos es muy interesante. Más detalles en [18].

${ }^{11}$ La Tc máxima reconocida por consenso es de un compuesto del YBCO bajo presión, Tc $=167 \mathrm{~K}$. Hay un sitio web, de acceso públi$\mathrm{co}$, que pretende haber logrado temperaturas muy superiores, superconductors.org, cuyos datos no son universalmente aceptados por la comunidad científica.
}

\subsection{Los Cupratos}

Dentro de la familia de los superconductores de alta Tc, los cupratos ocupan un sitio muy importante, no solo porque el primer material descubierto es un cuprato [8], sino también porque el primero con Tc mayor al nitrógeno líquido [9] fue, justamente, el YBCO, otro cuprato, quizás, el más estudiado de todos. Me voy a referir a este material.

Lo primero que se puede destacar es la complejidad de la estructura cristalina comparada con la simpleza de la de los superconductores convencionales. Observemos la Figura 8. Este tipo de estructuras reciben el nombre de perovsquitas. La estructura es casi tetragonal y hay varios trabajos teóricos en la literatura que lo tratan como tal, pero, estrictamente hablando, es ortorrómbica (grupo espacial Pmmm). Las medidas de sus lados son $a=3.8231, b=3.8864, c=11.6807$ Á (Angstroms ${ }^{12}$ ). Los átomos están identificados en la Figura 8. Se trata de la estructura estequiométrica. Una de las características de este material es que tiene varios conjuntos de átomos donde los electrones tienen un comportamiento diferente. Se les denomina escenarios. Las cadenas de $\mathrm{CuO}$, los planos de $\mathrm{CuO}_{2}$ y el eje-c. Los planos de cobre son los más importantes ya que ahí se forman los Pares de Cooper (y, posiblemente, en las cadenas). Las distintas familias se pueden diferenciar por el número de planos de $\mathrm{CuO}_{2}$. Un tratado muy completo y relativamente reciente es la ref. [26]. El diagrama de fases aparece en la siguiente Figura 9.

La familia del YBCO, comienza con un material que no tiene oxígeno en las cadenas, el $\mathrm{YBa}_{2} \mathrm{Cu}_{3} \mathrm{O}_{6}$. Por encima de una cierta temperatura de Neel, es dieléctrico, por debajo es antiferromagnético. El antiferromagnetismo yace en los planos de $\mathrm{CuO}_{2}$ (Ver Figura 8). A medida que la muestra va ganando oxígeno (éste se deposita en las cadenas) emigran electrones de los planos de $\mathrm{CuO}_{2}$, a través del oxígeno apical (ver Figura 8) hacia las cadenas de CuO. De esta manera, se abren huecos en los planos de $\mathrm{CuO}_{2}$. Estos huecos actúan como portadores de carga positiva (ausencia de un electrón en una banda llena). Y los pares de Cooper que se forman en esos planos son de portadores positivos. Desde una cierta concentración de oxígeno, la muestra se vuelve metálica, después de haber pasado por una fase de vidrio de espín (Ver Figura 9). Un poco después de volverse metálico el sistema, se vuelve superconductor. La temperatura máxima $(92 \mathrm{~K})$ la adquiere para $\mathrm{YBa}_{2} \mathrm{Cu}_{3} \mathrm{O}_{6.94}$, poco antes de la composición estequiométrica $\mathrm{YBa}_{2} \mathrm{Cu}_{3} \mathrm{O}_{7}(87 \mathrm{~K})$. El dopaje ulterior de oxígeno disminuye la temperatura crítica hasta que la superconductividad desaparece. La cantidad de datos experimentales sobre este material es enorme. En los últimos años, se ha intensificado el estudio de las interfaces de

\footnotetext{
${ }^{12} 1 \mathrm{~A}=10^{-8} \mathrm{~cm}$.
} 


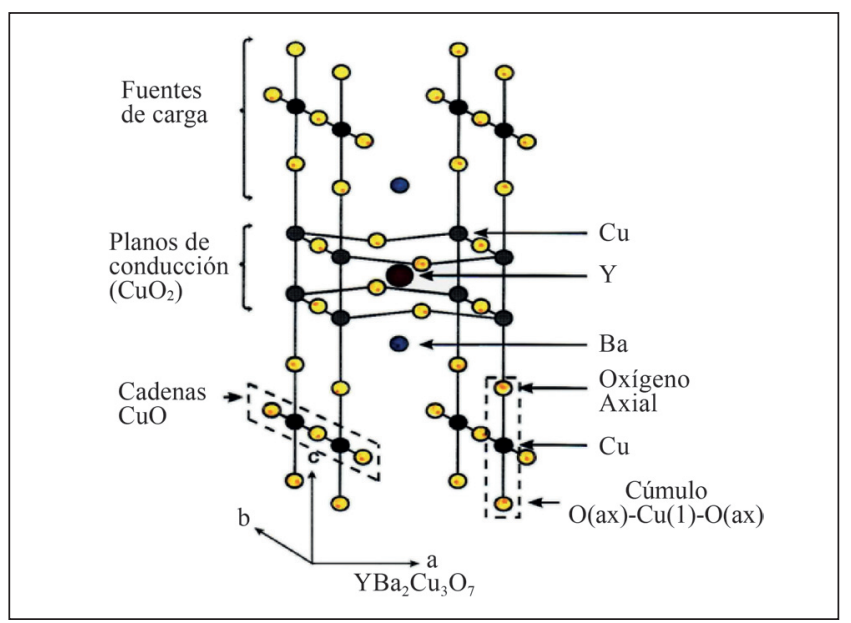

Figura 8. La estructura cristalina del YBCO7

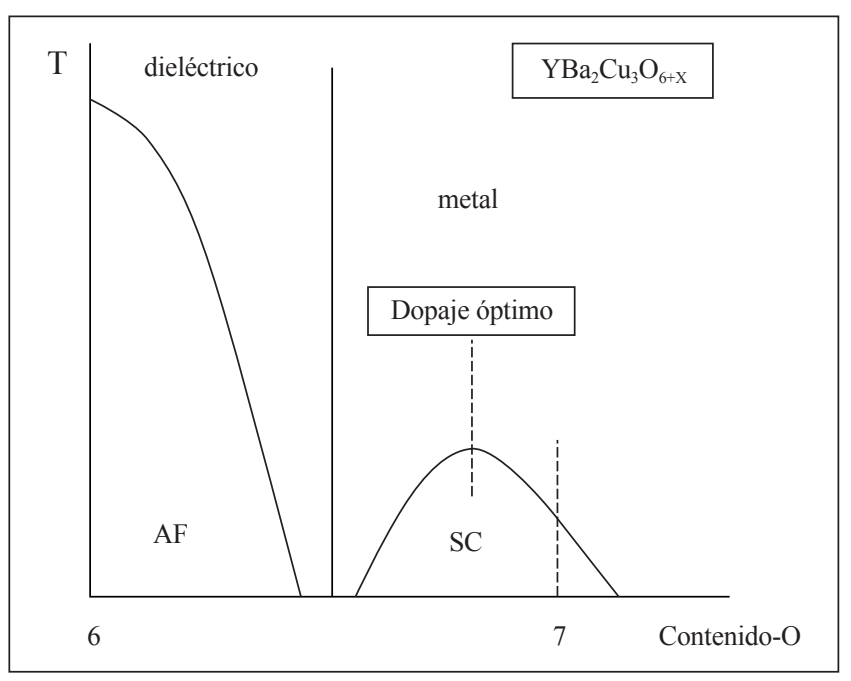

Figura 9. El diagrama de fases del $\mathrm{YBCO} 7$

este material (sobre todo en situación estequimétrica) con diversos materiales. De especial interés es la interface con semiconductores. Nosotros hemos estudiado la interface $\mathrm{YBa}_{2} \mathrm{Cu}_{3} \mathrm{O}_{7} /$ GaAs $[27,28]$ con el sorprendente resultado de que las capas del semiconductor adyacentes al $\mathrm{YBa}_{2} \mathrm{Cu}_{3} \mathrm{O}_{7}$, se vuelven metálicas. Esto permite esperar que la superconductividad se induzca en el semiconductor, permitiendo que la tecnología de los nanocircuitos con compuestos semiconductores, aproveche las propiedades propias del estado superconductor, especialmente, la no generación de calor.

\subsection{Algunas propiedades de estos superconductores}

El estado superconductor, más que un conjunto de pares de Cooper no relacionados entre sí, es un estado macroscópico coherente. Esto quiere decir que se necesita un volumen mínimo para que la transición de fase al estado superconductor sea posible. Este volumen se caracteriza por medio de la llamada longitud de coherencia que puede pensarse como la longitud característica del par de Cooper (como la distancia entre los dos electrones que constituyen el par de Cooper). Entre más grande sea, más alejado puede estar un electrón del otro con el que forma el par. Entre más grande sea esta distancia, podemos pensar que el "amarre" es más débil. En los superconductores convencionales esta distancia (que depende de la temperatura) es del orden de algunos cientos de angstroms a $\mathrm{T}=0 \mathrm{~K}$, pero puede llegar, como en el $\mathrm{Al}$ $(\mathrm{Tc}=1.2 \mathrm{~K})$, hasta los $1000 \AA \hat{\text {. }}$. De lo anterior se deduce que la longitud de coherencia es inversamente proporcional tanto a la brecha, $\Delta_{0}$, y a la Tc. La fórmula es, en efecto,

$$
\xi_{0}=\frac{2 \hbar v_{F}}{\Delta_{0}}
$$

donde $\mathrm{v}_{\mathrm{F}}$ es la velocidad de Fermi y el sub-índice 0, indica que el valor está tomado a $\mathrm{T}=0 \mathrm{~K}$. La longitud de coherencia de los nuevos materiales es muy inferior, es de decenas de

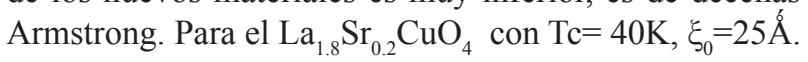

La brecha. Uno puede preguntarse, dada la pequeña longitud de coherencia y dada la existencia de escenarios en estos materiales como el YBCO, si, en realidad, existe una brecha y una Tc para cada escenario. Veamos el panorama. La conductividad a lo largo de las cadenas es casi el doble de la que se mide en la dirección perpendicular [29]. El tercer escenario (el eje-c) es metálico [30, 31, 32]. La diferencia, sin embargo, es que los electrones a lo largo del eje-c, permanecen en estado normal, por debajo de la Tc. Esto es algo nuevo, algo muy peculiar. Los dos escenarios, planos y cadenas, tienen la misma temperatura crítica, es un hecho experimental. Pero podrían tener dos brechas diferentes. Hay, en efecto, algunas evidencias experimentales acerca de la existencia de dos brechas. Por ejemplo, mediciones de la dependencia con la temperatura del desplazamiento Knight [40] y del tiempo de relajación de la resonancia nuclear magnética $[33,34]$ se encontró, en ambos casos, una función diferente cuando la medida se hacía sobre los planos y sobre las cadenas. Se encontró, además, que la relación entre la brecha y la temperatura crítica, Ec. 13, es del orden de 5 para los planos (mayor que BCS) y de 1.8 para las cadenas (mucho menor que BCS) [35]. De interés a este respecto es la ref. [36] donde, partiendo de la hipótesis de la existencia de dos valores de la brecha, se explican varios experimentos de manera coherente.

Esta es una breve descripción de las propiedades más sobresalientes de los cupratos, materiales sobre los cuales hay una gran actividad de investigación tanto teórica como experimental aun hoy (noviembre 2014). Una de las incógnitas principales es el origen, el mecanismo, que da lugar a la transición de fase superconductora. Más abajo abordaré 
el tema no sin advertir que no hay consenso acerca de ese punto que, junto con la predicción de la Tc, sigue como tema abierto y de gran interés.

\subsection{El diboruro de Magnesio.}

La estructura cristalina del diboruro de magnesio, $M g B_{2}$, aparece en la Figura 10.

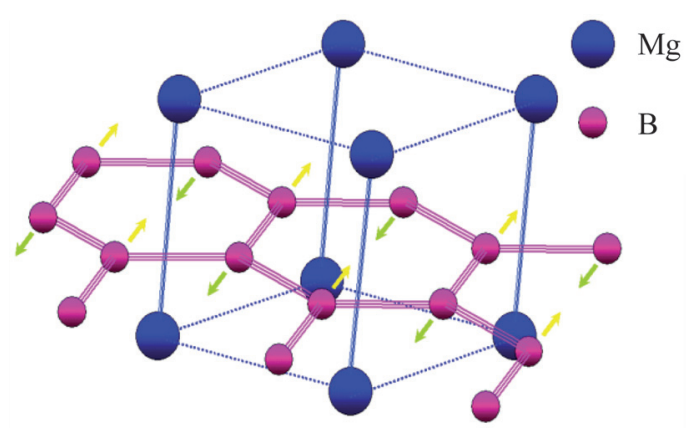

Figura 10. La estructura del diboruro de Magnesio

Es el superconductor de mecanismo electrón-fonón con la temperatura crítica más elevada que se conoce $(\mathrm{Tc}=40 \mathrm{~K})$, casi el doble del récord anterior a 1986 , del $\mathrm{Nb}_{3} \mathrm{Ge}$ con $\mathrm{Tc}=23 \mathrm{~K}$ (ver la ref. 23 acerca de ese material). Como puede verse, la estructura cúbica de los átomos de magnesio, sostiene una estructura hexagonal formada por átomos de Boro. Los pares de Cooper se forman en la estructura hexagonal debido a la atracción que suple un fonón en ese plano, las vibraciones de la red que dan lugar a ese fonón están marcadas con flechas en la figura. Se trata, por lo tanto, de un superconductor, esencialmente, bi-dimensional y anisotrópico.

Se observó dos brechas, 1.6 y $6.8 \mathrm{meV}$. La causa podría ser que el fonón involucrado es altamente anarmónico y es posible que se acople de distinta forma a diferentes grupos de electrones. Se trata de un superconductor de tipo II, su longitud de coherencia es de $4.4 \mathrm{~nm}$ (nanómetro ${ }^{13}$ ) a $\mathrm{T}=0 \mathrm{~K}$, La superconductividad en este material fue descubierta en el 2001. Lo curioso es que este material se conocía desde la década de los cincuentas y se vendía comercialmente. Pero nadie le midió la Tc, antes del 2001. Su estudio sigue activo (noviembre 2014)

\section{Superconductividad y magnetismo.}

La coexistencia entre superconductividad y magnetismo es un tema apasionante. Tiene varios aspectos. Las fluctuaciones de espín, por ejemplo, pueden actuar como un mecanismo que destruye la superconductividad electrón-fonón [37] pero también ha sido invocada como un mecanismo posible en

${ }^{13} 1 \mathrm{~nm}=10^{-9} \mathrm{~m}$ los nuevos materiales superconductores de alta Tc (ver más abajo). En esta sección trataré, brevemente, dos aspectos importantes de este tema, Uno la superconductividad en materiales que contienen hierro y el otro el comportamiento re-entrante.

\subsection{Los compuestos con Hierro (pnictides, en inglés)}

Hasta cierto punto, los compuestos con hierro son inesperados ya que una impureza magnética rompe totalmente la superconductividad electrón-fonón [37]. Sin embargo, en febrero del 2008 se anunció la existencia de un superconductor con Hierro, el $\mathrm{LaO}_{1-\mathrm{x}} \mathrm{F}_{\mathrm{x}} \mathrm{FeAs}$ con $\mathrm{Tc}=26 \mathrm{~K}$, y una estructura laminar (ver Figura 11), formada por planos de $\mathrm{LaO}$ y de FeAs. La familia, los oxinitrogenuros, son estructuras tetragonales, laminares, de fórmula general (Figura 11a) LnOTmPn, donde $\mathrm{O}$ es oxígeno, $\mathrm{Ln}=\mathrm{Ce}, \mathrm{Pr}, \mathrm{Nd}, \mathrm{Sm}, \mathrm{Th}, \mathrm{Dy} ; \mathrm{Tm}$ es un metal de transición y $\mathrm{Pn}=\mathrm{P}, \mathrm{As}, \mathrm{Bi}$. La otra familia es la de los arseniuros ternarios de fórmula general (Figura 11b) $\mathbf{A F e} \mathbf{A s}_{2} \mathbf{A s}$, donde $\mathrm{A}=\mathrm{Ca}, \mathrm{Sr}, \mathrm{Ba}$. En estos materiales la $\mathrm{Tc}=26-55 \mathrm{~K}$.

La investigación de estos materiales está muy activa e, incluso, ciertas características cristalinas llaman la atención. Una de ellas es que la Tc depende de la constante de red del material con diferentes Ln, como se ve en la siguiente Figura 12. La síntesis misma de estos materiales es objeto de investigación todavía.

En la fase no-superconductora del LaOFeAs (compuestos nodopados) se ha encontrado, por difracción de neutrones y de rayos $\mathrm{X}$, una transición de fase estructural a $\mathrm{T}=155 \mathrm{~K}$ de una estructura tetragonal a otra ortorrómbica. Posteriormente, a $\mathrm{T}=137 \mathrm{~K}$, la aparición de un orden magnético de largo alcance, es causa de una transición antiferromagnética en los átomos de $\mathrm{Fe}$. $\mathrm{Al}$ dopar los materiales, estas transiciones se suprimen y solo permanece la transición superconductora. Por otro lado, se ha encontrado también que la Tc depende de un cierto ángulo de la estructura. Este es otro aspecto sin explicación cabal en estos complejos materiales que son actualmente tema de intensa investigación.

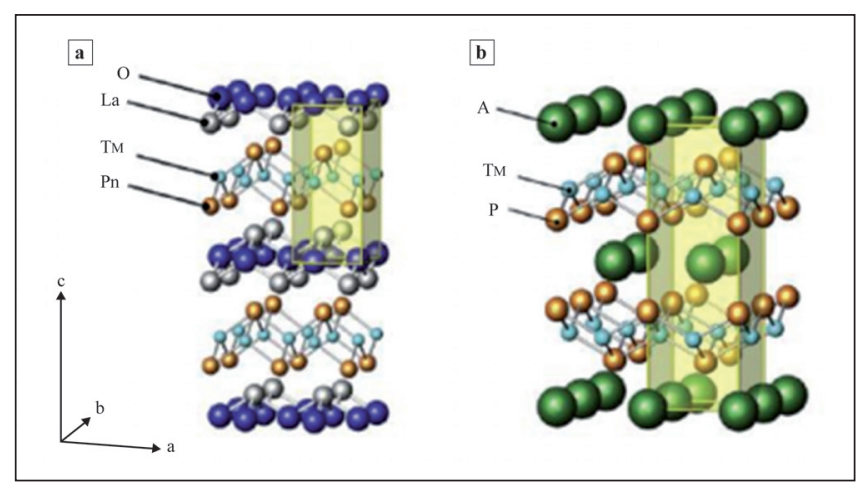

Figura 11. Estructura cristalina superconductores con Fe 


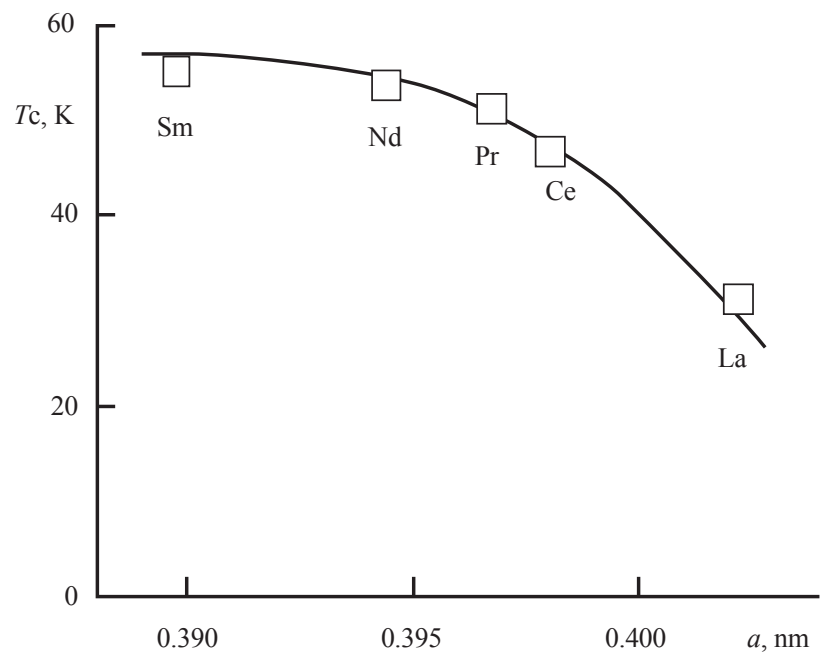

Figura 12. Relación entre el elemento usado en el compuesto LnOTmPn que determina una constante de red del material y la temperatura crítica. Esta dependencia no está explicada.

\subsection{El comportamiento re-entrante}

Comportamiento re-entrante se llama al hecho de que, por debajo de la temperatura crítica superconductora un material regrese espontáneamente al estado normal. Hay tres tipos diferentes de comportamiento re-entrante. En los dos primeros hay presencia de Tierras Raras en el material que forman, a una cierta temperatura de Neel, $\mathrm{T}_{\mathrm{N}}$, una sub-red magnética que colapsa el estado superconductor, regresando la muestra al estado normal. El segundo tipo, se da en muestras en las cuales, a una temperatura aun menor que la de Néel, la superconductividad reaparece. El tercer tipo de comportamiento re-entrante se presenta en compuestos donde no hay Tierras Raras. Cuando el compuesto tiene Tierras Raras, como en los Borocarburos del tipo $\mathrm{RT}_{2} \mathrm{~B}_{2} \mathrm{C}$ donde $\mathrm{R}$ es una Tierra Rara ( $\mathrm{Sc}$, $\mathrm{Y}, \mathrm{La}, \mathrm{Ho}, \mathrm{Er}$, por ejemplo), y T= Ni, Pd, Ru, por ejemplo, la sub-red de la Tierra Rara se vuelve antiferromagnética a $\mathrm{T}_{\mathrm{N}}<$ $\mathrm{T}_{\mathrm{c}}$. Por esa razón, la muestra pasa al estado superconductor $\mathrm{y}$, al descender la temperatura hasta la temperatura de Neel, el orden antiferromagnético da cuenta de la superconductividad, como ya lo mencioné [38]. El fenómeno fue descubierto hacia los años setentas. Presento aquí, un descubrimiento hecho en 1993, una muestra donde la superconductividad reaparece a una temperatura menor a la de Néel, como se ilustra en la Figura 13. En algunos casos la superconductividad no reaparece. Este comportamiento re-reentrante es un problema abierto, sin explicación aun.

Un problema diferente, aparentemente, es el comportamiento que presentan los compuestos que no contienen tierras raras, Un caso de este tipo fue reportado para el experimento de dos monocristales intercalados entre sí [39], $\mathrm{Bi}_{2} \mathrm{Sr}_{2} \mathrm{CaCu}_{2} \mathrm{O}_{8}$ ' $\mathrm{Bi}_{2} \mathrm{Sr}_{2} \mathrm{Ca}_{2} \mathrm{Cu}_{3} \mathrm{O}_{10}$. Ante una corriente pequeña de $50 \mu \mathrm{A}$, la resistencia del sistema de cristales intercalados entre sí, pasa al estado superconductor a $105 \mathrm{~K}$ y regresa a un estado de resistencia finita (estado normal) a una temperatura inferior para después volver al estado superconductor (resistencia cero) a los $92 \mathrm{~K}$. No hay tampoco explicación convincente para este experimento. El modelo que los autores usan para explicarlo es un sistema compuesto por un superconductor en interface con un semiconductor.

En un estudio posterior [40] se fabricó un intercalado de micropartículas de $\mathrm{CdS}$ dentro de una cerámica de $\mathrm{Bi}-\mathrm{Pb}-\mathrm{Sr}-\mathrm{Ca}-$ $\mathrm{Cu}-\mathrm{O}$. El material base es el Bi-2223 $\left(\mathrm{Bi}_{2} \mathrm{Sr}_{2} \mathrm{Ca}_{2} \mathrm{Cu}_{3} \mathrm{O}_{10}\right)$. El resultado se ilustra en la Figura 14. Aparecen dos curvas que corresponden a una medida de resistencia contra temperatura

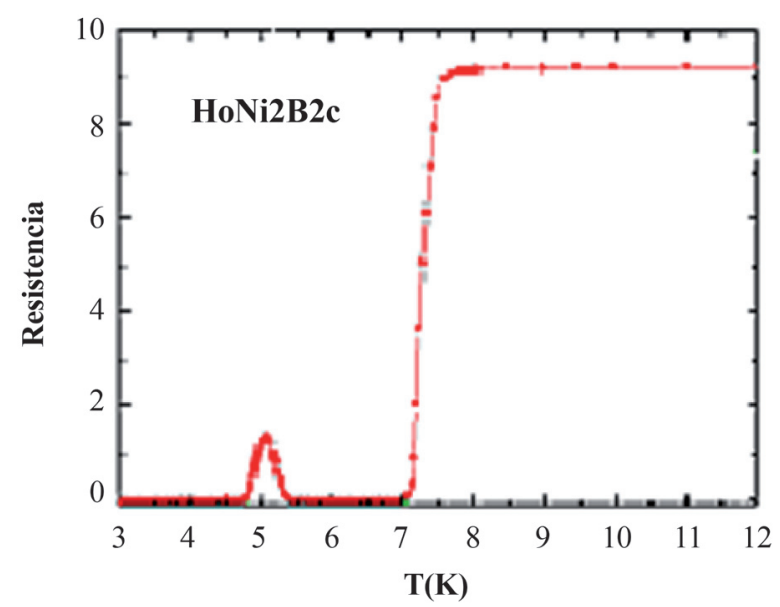

Figura 13. Resistencia vs. Temperatura. Descubierto por R. Cava en 1993 en el borocarburo $\mathrm{HoNi}_{2} \mathrm{~B}_{2} \mathrm{C}$, este intrigante comportamiento re-re-entrante se ha encontrado en varias muestras del mismo tipo y sigue aun sin explicación. La muestra regresa al estado superconductor a una temperatura por debajo de la $\mathrm{T}_{\mathrm{N}}$.

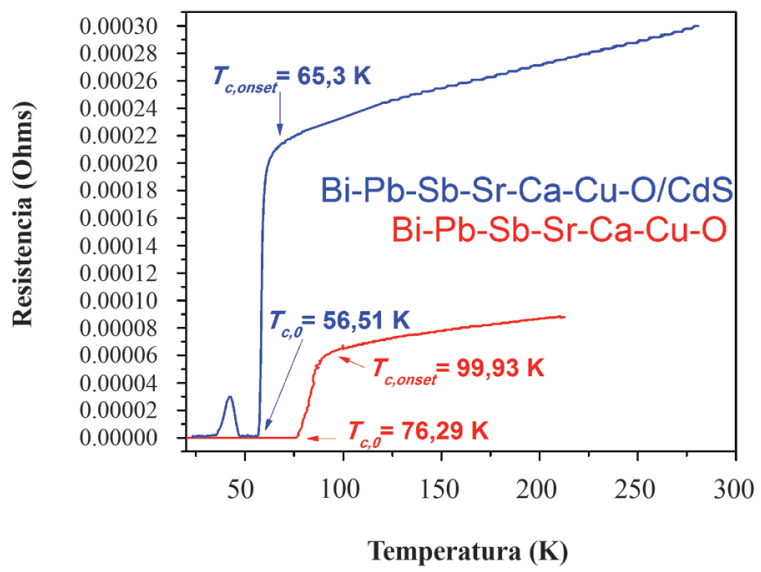

Figura 14. El comportamiento re-re-entrante en un composito intercalado con micro-partículas de CdS. 
de la cerámica sola (curva inferior) y del composito (curva superior). La cerámica comienza a bajar su resistencia hacia los $94 \mathrm{~K}$ y registra resistencia cero a los $76 \mathrm{~K}$, el ancho de la transición es de $18 \mathrm{~K}$. Permanece en el estado superconductor a toda temperatura por debajo de los $76 \mathrm{~K}$. La curva superior es una medida de la resistividad del composito. Su resistencia hacia los $200 \mathrm{~K}$ es del orden de tres veces mayor que la de la cerámica sola. El composito comienza la transición a una temperatura igual a los $65 \mathrm{~K}$ y el valor cero de la resistencia se mide a los $56 \mathrm{~K}$, la transición es de $9 \mathrm{~K}$, mucho menor que la de la cerámica sola. El composito pasa al estado normal por debajo de los $50 \mathrm{~K}$ y regresa al estado superconductor por encima de los $40 \mathrm{~K}$. En este caso no hay Tierras Raras. El fenómeno requiere una explicación diferente a la formación de una sub-red antiferromagnética. Otro problema abierto.

\section{El mecanismo.}

Ha habido una búsqueda tenaz del mecanismo. La interacción electrón-fonón fue presentada por el grupo de Berkeley como un posible mecanismo al observar que en el YBCO7, un fonón cercano a los $80 \mathrm{meV}$ se ablandaba (se corría hacia frecuencias menores) a la temperatura de la transición superconductora [41]. Una reinterpretación del experimento [42] mostró que las fluctuaciones de espín podrían explicar el experimento de igual manera. Las fluctuaciones de espín, si tienen que ver con el mecanismo tienen que mostrarse en experimentos de susceptibilidad magnética. Esta función presenta una resonancia a $40 \mathrm{meV}$ en YBCO7, la cual fue utilizada por Carbotte et al. [43] como la función de Eliashberg para calcular, usando las Ecuaciones de Eliashberg, la termodinámica del YBCO7. Sin embargo, un audaz experimento demostró que la hipótesis era incorrecta [44 ]. Este es el reporte de uno de los muchos intentos en esta dirección. El mecanismo sigue como un problema abierto.

\section{Conclusiones}

La superconductividad ha sido un campo muy fértil que ha influido el desarrollo tanto teórico como experimental en muchas áreas de la física incluyendo la teoría de Muchos Cuerpos y las partículas elementales. El fenómeno es cuántico macroscópico no relativista. Su interés se ha aumentado ante la posibilidad de múltiples aplicaciones tecnológicas. La teoría BCS (1957) estableció que la superconductividad es la física de los pares de Cooper los cuales se mantienen por interacción con la red (fonones). Esta abrió el camino para que una formulación más exacta basada en teoría de campo no-relativista, estableciera las ecuaciones de Eliashberg y lograra una explicación total y cabal del fenómeno. En 1986, Bednorz y Müller [45] descubrieron los superconductores de alta temperatura crítica, re-abriendo el campo desde cero. Los problemas que se planteaban en 1911, año en que K. Onnes descubrió la existencia de la superconductividad, se plantean aun hoy (noviembre 2014) para la nueva superconductividad no-convencional. Una rica serie de fenómenos nuevos se presentan, y una búsqueda tenaz para entenderlos hace de esta disciplina una de las más interesantes y activas entrada ya la segunda década del siglo XXI. Un gran problema abierto por encima de todos: predecir la temperatura crítica desde primeros principios. Registro, por completez, los trabajos del grupo de Gross [46] a los cuales no me referí por falta de espacio. Tampoco me referí a "grafeno, grafito y superconductividad" que es un tema en estudio de gran interés por las propiedades muy novedosas del grafeno. Hay superconductividad para otro siglo más, al menos. Y pensar que, a comienzos de 1986, creíamos que todo estaba terminado para la superconductividad.

\section{Referencias}

1. Onnes K. (1911) Further experiments with liquid helium. On the change of electric resistance of pure metals at very low temperatures, etc. IV. The resistance of pure mercury at helium temperatures. Comm. Phys. Lab. Univ. Leiden 29, 1 (1911).

2. Baquero R., (1997) El Descubrimiento de la Superconductividad, Avance y Perspectiva (Cinvestav), 16, 167.

3. Frölich H., (1950) The Isotope Effect in Superconductivity, Procc. Phys. Soc. A63, 778.

4. Cooper L.N. (1956), Bound Electron Pairs in a Degenerate Fermi Gas, Phys. Rev. 104, 1189.

5. Bardeen J., Cooper L.N. and Schrieffer J.R., (1957), Microscopic Theory of Superconductivity, Phys. Rev. 106, 162.

6. Eliashberg G.M., (1960) Interactions Between Electrons and Lattice Vibrations in a Superconductor, Soviet Phys. JEPT 11, 696.

7. Narrado por él mismo durante una reciente visita al IPN, México.

8. Bednorz J. G. and Müller, K.A. (1986) Possible High-Tc Superconductivity in the Ba-La-Cu-O System, Z. Phyzik B 64, 189.

9. Chu C.W. et al., (1993) Superconductivity above $150 \mathrm{~K}$ in $\mathrm{HgBa}_{2} \mathrm{Ca}_{3} \mathrm{O}_{8+\delta}$, Nature 365, 323.

10. Baquero R., (2005) Brief Introduction to Superconductivity, 1 Edición, Editado por el Departamento de Física, Cinvestav. México, D.F.

11. Baquero R., (2006) El Fascinante mundo del Estado Sólido: La superconductividad, 1 Edición, editado por la Universidad Pedagógica y Tecnológica de Colombia (UPTC), Tunja, Colombia.

12. Navarro O. y Baquero R., (2007) Ideas fundamentales de la Superconductividad, 1 Edición, Editado por la Universidad Nacional Autónoma de México (UNAM), México D.F.

13. Fernandes R.M. (2012), AIP Conf. Proc. 1420, 135.

14. Shrodinger E., (1926) Quantisierung als Eigenwertproblem, Annalen der Phyzik (4) 79, 489. 
15. N. Bohr es autor del primer modelo atómico que incluía una cierta forma de cuantización. Es autor de una interpretación de la Mecánica Cuántica llamada la interpretación de Copenhage que, aunque discutida, se impuso en la mayoría de los físicos del Siglo XX y prevalece hasta nuestros días.

16. A. Einstein y N. Bohr tuvieron una famosísima polémica que nunca concluyó acerca de la interpretación de la Mecánica Cuántica. Einstein no estuvo de acuerdo con la interpretación probabilísca de Bohr. "Dios no juega a los dados", parece haber dicho.

17. Fetter A. and Walecka J.D., (1971) Quantum Theory of ManyParticle systems, $1^{\text {st }}$ Edition, Mc Graw Hill Editors, Mexico; Mahan G.D., (1981) Many Particle Physics, $1^{\text {st }}$ Edition, Plenum Press, New York and London; Schrieffer J.R., (1964), Theory of Superconductivity, W.A. Benjamin, INC. Reading, Ma. USA

18. Kittel C., (1996) Introduction to Solid State Physics J. Wiley \& Sons, Inc. New York:; Kittel C., (1996) The Quantum Theory of Solids; W. A. Harrison (1996), Electronic Structure and the Properties of Solids.

19. G. Rickayzen G. (1996), Theory of Superconductivity, Wiley, New York; J.R. Schrieffer, (1965) Theory of Superconductivity, Addison-Wesley Publishing Company Inc; R. Parks editor, (1969) Superconductivity, Marcel Dekker, N.Y.; G. Burns, (1992) High-Temperature Superconductivity: An Introduction, Academic Press, Inc., , entre otros muchos.

20. Revisiones de la teoría de Eliashberg se encuentran en D.J. Scalapino (1969), Superconductivity, editado por E.D. Parks, Marcell Dekker, New York, vol. 1, pág. 449; Allen P.B. and Mitrovic B., (1982) Solid State Physics, editado por F. Seitz, D. Turnbull, y H. Ehrenreich, Academic Press, N.Y. vol 37 pág. 1; Carbotte J.P., (1990) Electron-Boson Superconductivity, Rev. Mod. Phys. 62, 1027.

21. Daams J.M., J.P. Carbotte, J.P. and Baquero R. (1979), Critical Field and Specific Heat of Superconducting Tl-Pb-Bi Alloys, J. Low Temp Phys. 35, 547.

22. Abrikosov A. A. and Gorkov,L.P., (1961), Contribution to the Theory of Superconducting alloys with paramagnetic impurities. Soviet Physics JEPT, 12,1243.

23. Baquero R., Gutierrez-Ibarra J., Meza L., Navarro O., and Kihlstrom K.F., (1989) Eliashberg Theory and High-Tc Superconductiity, Rev. Mex. Phys. 35, 461 .

24. V.L. Ginzburg and L.D. Landau, Zh. Eksp. Teor. Fiz. 20, 1064 (1950); L.D. Landau (1965) Collected Papers, Oxford Pergamon Press, 1965, pág. 546. La teoría de transiciones de fase de Ginsburg-Landau es una de las aportaciones más importantes de la física del siglo XX. Se aplica a la superconductividad y coincide con BCS en temperaturas cercanas a la transición de fase. Una breve revisión de la misma se encuentra en la ref. 12.

25. Abrikosov A. A., (1957), On the magnetic propeties of superconductors of the second group, Soviet Phys. JETP 5, 1174.
26. Poole C.P., (2000) Handbook of Superconductivity, Academic Press, USA.

27. A.E. García et al., (2008), Influencia de la temperatura en la estructura de bandas. R. Baquero-Salaquarda, A.E. García, L.M. García-Cruz, and R. Baquero, The ICFAJ Univ. J. Phys. 1, 28.

28. R. Torres and R. Baquero, (2013) Metallic behavior of the YBCO7/ZAl interfaces ( $Z=G a, A s)$. Rev. Méx. Fís. 59 493-497.

29. Friedman T. et al., (1990), Direct measurement of the anisotropy of the resistivity in the plane of twin-free, singlecrystal superconductivity, Phys. Rev. B 42, 6217.

30. Rubio-Ponce A., (1999), Escenarios activos de los materiales superconductores. PhD. Thesis, Cinvestav, México D.F.

31. Rubio-Ponce A. and Baquero R., (2001) Superconductividad en $\mathrm{YBa}_{2} \mathrm{Cu}_{3} \mathrm{O}_{7}$ ¿Un fenòmeno bidimensional? Rev. Mex. Fís. 47, 447 .

32. Puch F., (2003) Hacia el mecanismo de los nuevos superconductores: La importancia de la tridimensionalidad. PhD. Thesis, Cinvestav, México D.F.

33. Barret S. et al., (1990), ${ }^{63} \mathrm{Cu}$ Knight shift in the supeconducting state of $\mathrm{YBa}_{2} \mathrm{Cu}_{3} \mathrm{O}_{7-\delta}$ Phys. Rev. B 41, 6283.

34. Waren W. et al., (1987) Evidence for two pairing energies from Nuclar spin-lattice relaxation in superconducting $\mathrm{Ba}_{2} \mathrm{YCu}_{3} \mathrm{O}_{7-\delta}$ Phys. Rev. Lett., 591860.

35. F, Puch and R. Baquero, (2005) The optical conductivity resonance from an exact description of the electronic states around the Fermi energy. arXiv cond-matt/0504593.

36. Cucolo A.M, Noce C. And Romano A., Simulation of experimental results on high-Tc superconductors: A phenomenological model, Phys. Rev B 536764.

37. Baquero R., Daams J.M. and Carbotte J.P. (1981), Detailed study of the thermodynamics of a superconductor with paramagnons, J. Low Temp. Phys. 35, 585.

38. Muller K.H. and Narozhnyi V.N., (2001) Interaction of superconductivity and magnetism in borocarbide superconductors, Rep. Prog. Phys. 64, 943.

39. Zhao Y. et al., (1995), Grating-induced cyclotron resonance anomaly in GaAs/ $\mathrm{Al}_{\mathrm{x}} \mathrm{Ga}_{1-\mathrm{x}}$ As heterostructures, Phys. Rev. B 51,

40. A.E. Valdés et all. (2011) Processing and study of the composite $\mathrm{CdS} / \mathrm{Bi}-\mathrm{Pb}-\mathrm{Sr}-\mathrm{Ca}-\mathrm{Cu}-\mathrm{O}$, arXiv cond-mat/ 1101.0277

41. A. Lanzara A. et al. (2001), Evidence for ubiquitous electronphonon coupling in high-temperature superconductors, Nature 412, 510 .

42. Schachinger E., Tu J.J. and Carbotte J.P., (2003) Superconducting-insulating transition in quantum three-dimensional Josephson junction arrays with magnetic and charge frustration, Phys Rev B 67, 214505.

43. Carbotte J.P., Schachinger E. and Basov E.D. (1999) Coupling strenth of charge carriers to spin fluctuations in hightemperature superconductors, Nature (London) 401, 354. 
44. Huang J.H, Timusk T., and Gu G. D.,(2004) High-transitiontemperature superconductivity in the absence of the magnetic resonance mode. Nature 427, 714

45. “The Nobel Prize in Physics 1987”. Nobelprize.org. Nobel Media AB 2013. Web. 25 Oct 2013. http://www.nobelprize. org/nobel_prizes/physics/laureates/1987/
46. Lüders M. et al. (2005) Ab initio theory of superconductivity. I Density Functional formalism and approximate functionals. Phys. Rev B 024545; Márques M.A. et al. (2005) Ab initio theory of superconductivity II Application to elementary metals, Phys. Rev B 024546. 